\title{
Changes of Ascorbic Acid Content in Leaves and Fruits of Ziziphus jujube'Zhanshanmizao 1'at Different Developmental Stages
}

\author{
Shuyue Huang ${ }^{1, a}$, Nengli Fu, ${ }^{2, b}$, Guoying Song ${ }^{3, c}$, and Huifen Zhang ${ }^{4, d *}$ \\ ${ }^{1}$ College of Horticulture, Sichuan Agricultural University, Chengdu, Sichuan, China \\ a1183328242@qq.com, b1530779913@qq.com, c344596038@qq.com, d1042335395@qq.com \\ ${ }^{*}$ Corresponding author.
}

\begin{abstract}
Keywords:Ziziphus jujube'Zhanshanmizao 1';ascorbic acid;change.
Abstract. In order to find out the accumulation of ascorbic acid in different tissues of Ziziphus jujube 'Zhanshanmizao 1', this experiment used Ziziphus jujube 'Zhanshanmizao 1'as the test material to determine the ascorbic acid content in different developmental stages and flowers of Ziziphus jujube 'Zhanshanmizao 1' fruit and leaves. The dynamic changes of ascorbic acid in leaves and fruits of Ziziphus jujube'Zhanshanmizao 1'were studied. The results showed that the ASA content of Ziziphus jujube 'Zhanshanmizao 1'fruit increased gradually from the young fruit stage to the white mature stage, reached its maximum value at the white mature stage, and then gradually decreased with the maturity of the fruit; however, the DHA content changed during the entire fruit development period. Not great. The content of ASA in leaves of Ziziphus jujube 'Zhanshanmizao 1' gradually decreased during the whole development period, while the content of DHA increased rapidly in the green period, peaked in the white mature stage, and then gradually decreased with the aging of the leaves. In the same period, the content of ASA in Ziziphus jujube 'Zhanshanmizao 1' was higher than that in leaves and flowers, and the content of DHA in leaves was the highest, and the total content of ascorbic acid was highest in leaves. Therefore, the accumulation rules of ascorbic acid in the fruits and leaves of Ziziphus jujube'Zhanshanmizao 1' were significantly different, and there were differences in the forms and contents of ascorbic acid in different tissues.
\end{abstract}

\section{Introduction}

Ziziphus jujuba'Zhanshanmizao 1' originates from Santai Village,Zhanshan County,Mianyang City,Sichuan Province. The fruit is thin, sweet and of good quality. It is also rich in ascorbic acid and is available on the market. Widely enjoyed by consumers ${ }^{[1-2]}$. L-ascorbic acid, also known as vitamin $\mathrm{C}(\mathrm{Vc})$, is a class of hexose lactone compounds synthesized in vivo. Total ascorbic acid consists of reduced ascorbic acid (ASA) and oxidized ascorbic acid (DHA). ASA is an indispensable and important substance for plant growth and development. It not only plays an important role in antioxidant capacity, cell division and elongation ${ }^{[3]}$, but also plays an important regulatory role in plant resistance ${ }^{[4-5]}$. Studies have shown that during the fruit development process of Ziziphus jujuba cv.Jinping, the content of ascorbic acid decreases first, then rises and then falls ${ }^{[6]}$; the content of ascorbic acid in Ziziphus jujuba cv.Jinsixiaozao fruit rises first and then decreases ${ }^{[8]}$; the content of ascorbic acid in Zizyphus jujuba Mill. cv. Lingwuchangzao leaves does not change in each period. Obviously ${ }^{[7]}$. Although there are some reports on the study of ascorbic acid in jujube in recent years, there are still few reports on the research of ascorbic acid in leaves and fruit of Ziziphus jujube 'Zhanshanmizao 1'. Therefore, this study intends to study the variation of ascorbic acid content in leaves and fruits of different developmental stages in the flower of Ziziphus jujube 'Zhanshanmizao 1 ' and compare the difference in ascorbic acid content between different tissues to provide basic data for the study of ascorbic acid synthesis and regulation mechanism in jujube.

\section{Materials and Methods}

Materials. The 7-year-old Ziziphus jujube 'Zhanshanmizao 1' with uniform tree growth and consistent growth was selected as the test tree. The planting density was $2 \mathrm{~m} \times 3 \mathrm{~m}$. Three plants were used in one 
plot and repeated three times.

Experimental Design.Sample flowers collection: The first and second batches of jujube were collected at flowering stage. Sample leaves collection: When the first batch of jujube flowers and thanks to fruiting, select the date of growth consistent with the date hanging for listing. From May 5 onwards, the sampling times were May 5 (young leaves), May 12 (young leaves), June 4 (expanding leaves), June 24 (expanding leaves), July 14 Days (matured leaves), August 3 (aging leaves), and August 23 (aging leaves); fruit collection: Starting from June 4th, sampling every 20 days until fruit ripening, sampling time is June respectively 4th (young fruit period), June 24 (green maturation period), July 14 (white maturation period), August 3 (transfection period), August 23 (red maturation period). Each sample was collected at 10:00 a.m. from the plant's east, south, west, north, top, and inner halls, and a uniform size of $\geq 30$ fruits and $\geq 50$ leaves were uniformly collected. Immediately after the harvest, the ice box was used to bring it back to the laboratory, and it was stored in a refrigerator at $-80^{\circ} \mathrm{C}$ until use. The indicators were repeatedly measured three times. Select 30 fruits with normal growth and development, and use the electronic balance to weigh the total weight of 30 samples, calculate the average fruit weight; use the digital display vernier calipers to measure the fruit's vertical diameter and transverse diameter according to the average of each observation record, and draw the fruit growth curve. Determination of reduced Ascorbic acid (ASA) and dehydro ascorbic acid (DHA) content in leaves and fruits. The Fe $3^{+}$reduction method of Kampfenkel ${ }^{[9]}$ was measured and adjusted slightly.

\section{Results and Discussion}

Dynamic Changes of Growth and Development of Jujube Fruits As can be seen from Fig.1, the transverse and longitudinal diameters of Ziziphus jujube 'Zhanshanmizao 1' increased rapidly from the young fruit stage to the green ripe stage, then increased slowly, and slightly decreased during the color change period. The single fruit weight increased rapidly in the young fruit period and the color conversion period, and then slowly increased. Taken together, the single fruit weight of jujube cultivars generally shows the trend of "S".

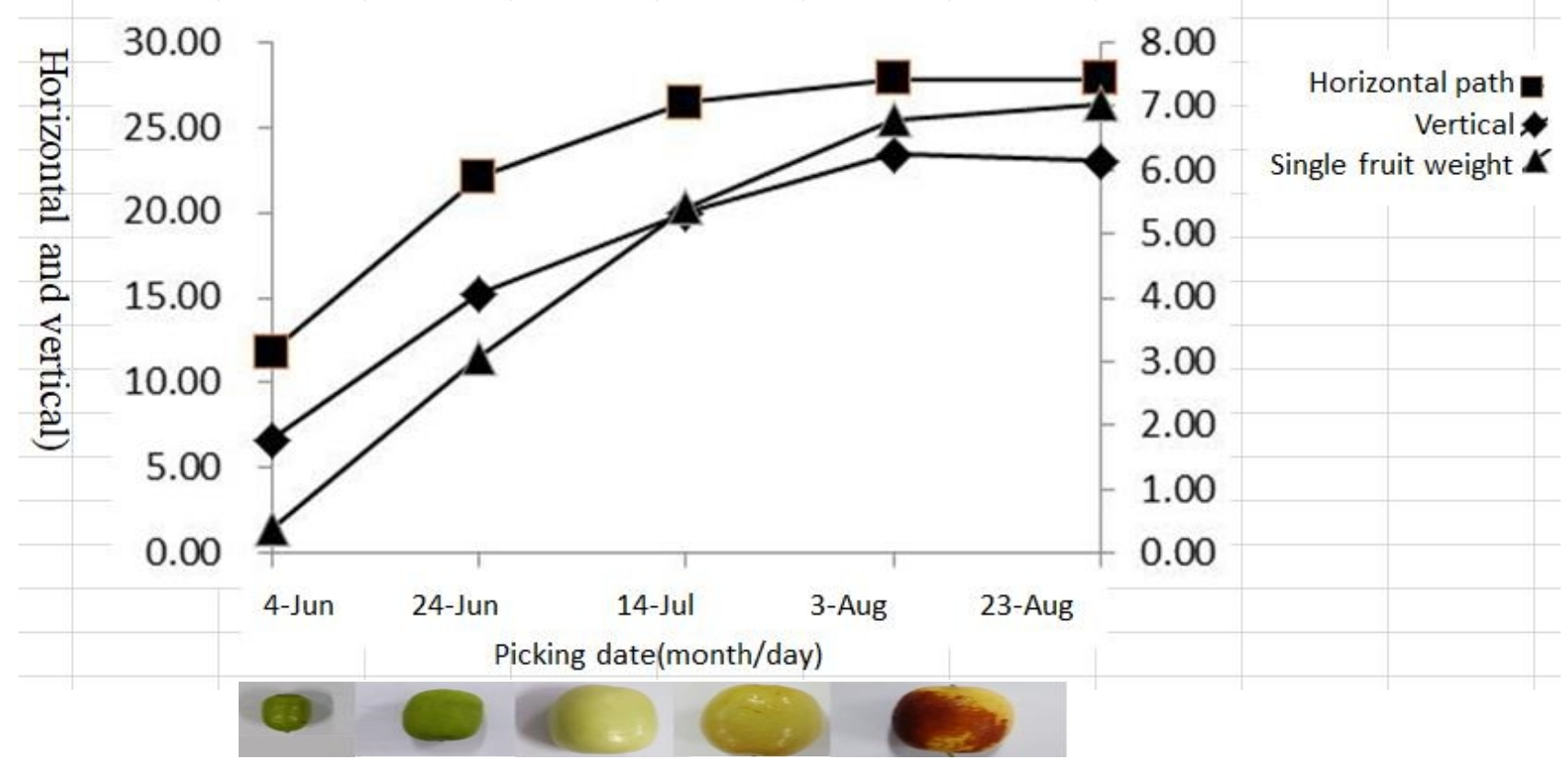

young fruit green maturation white maturation transfection red maturation

Fig.1,The growth,development and morphological changes of fruit of Ziziphus jujube 'Zhanshanmizao 1' 
Changes of ASA and DHA Contents in Chinese Jujube Fruits at Different Development Stages As can be seen from Fig.2, we can see that the content of ASA increased firstly and then decreased during the development of the fruit of Ziziphus jujube 'Zhanshanmizao 1'and reached its maximum at the white mature stage with a maximum value of $312.00 \mathrm{mg} / 100 \mathrm{~g} \cdot \mathrm{FW}$, which is a $17 \%$ increase compared to the young fruit stage. The DHA content decreased first, then rose and then decreased. It reached its maximum value in the color-changing period. The maximum value was 48.22 $\mathrm{mg} / 100 \mathrm{~g} \cdot \mathrm{FW}$, which was $20.55 \%$ higher than the young fruit period. During the whole growth period, the DHA content was lower than the ASA content. Taken together, ascorbic acid is predominantly present in the form of ASA in fruits, and changes in ASA and DHA show different trends, but there is a peak content.

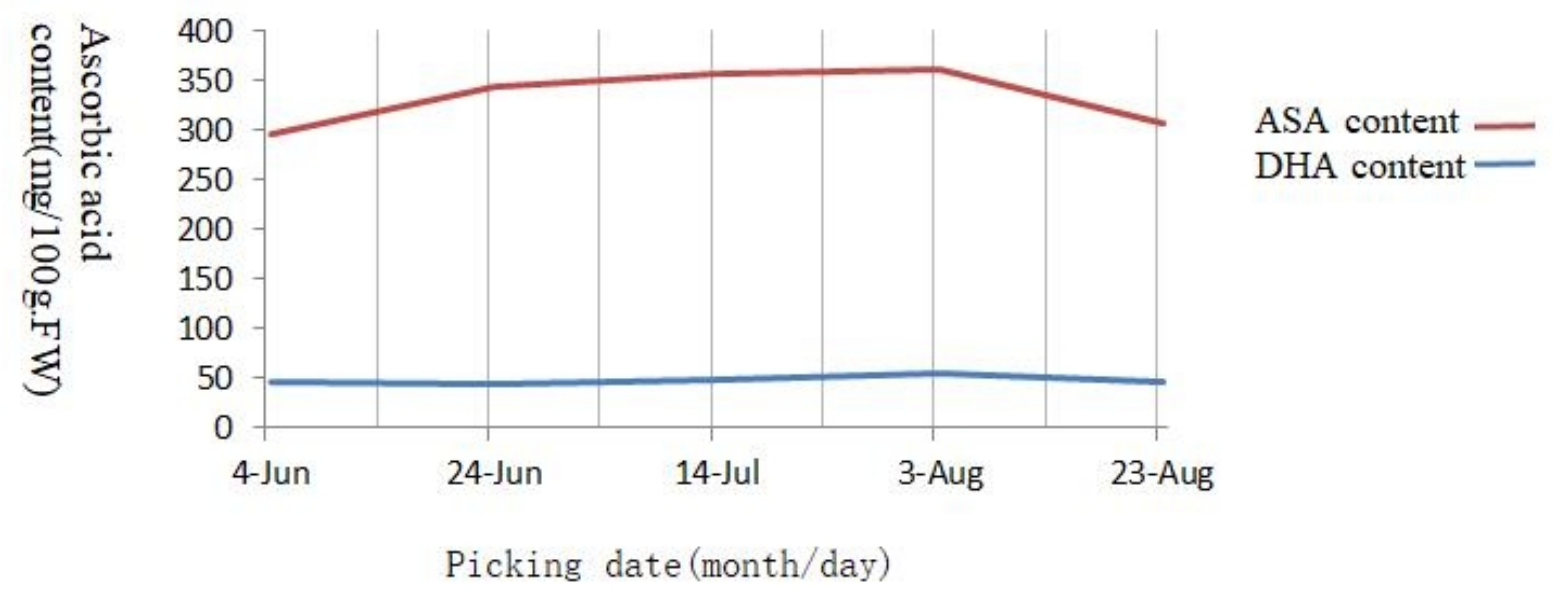

Fig.2, Changes of ASA and DHA Contents in the fruits of Ziziphus jujube'Zhanshanmizao 1'

Changes of AsA and DHA Contents in Leaves of Jujube at Different Growth Stages. As can be seen from Fig.3, it can be seen that the ASA content of the leaves of Ziziphus jujube 'Zhanshanmizao 1 ' continued to decline during its development, and the lowest value appeared in the senescence period, which was reduced by $64.21 \%$ compared to the young leaf stage; while the DHA content declined from the young leaf stage to the expanding leaf stage. Afterwards, it began to rise, reaching the highest at the mature leaf stage, which was $300 \mathrm{mg} / 100 \mathrm{~g} \cdot \mathrm{FW}$, which was an increase of $66.7 \%$ compared with the expansion leaf stage, and then began to decline. From Fig. 4, it can be seen that AsA/DHA content in leaves of Ziziphus jujube 'Zhanshanmizao 1' first rises, then decreses and then rises. The ASA/DHA ratio reached the highest during the spreading leaf period, then began to decline, and then rose again in the mature leaf period. Taken together, during the development of the leaf, ascorbic acid mainly exists in the form of ASA in the early stage, but it mainly exists in the form of DHA in the later stage.

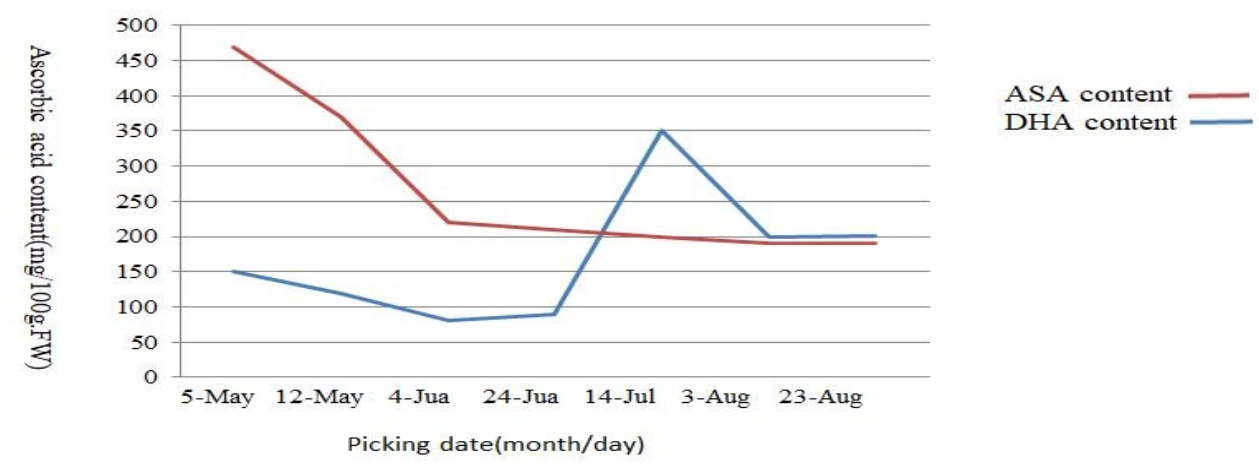

Fig.3, Changes of ASA and DHA content in leaves of Ziziphus jujube 'Zhanshanmizao 1' 


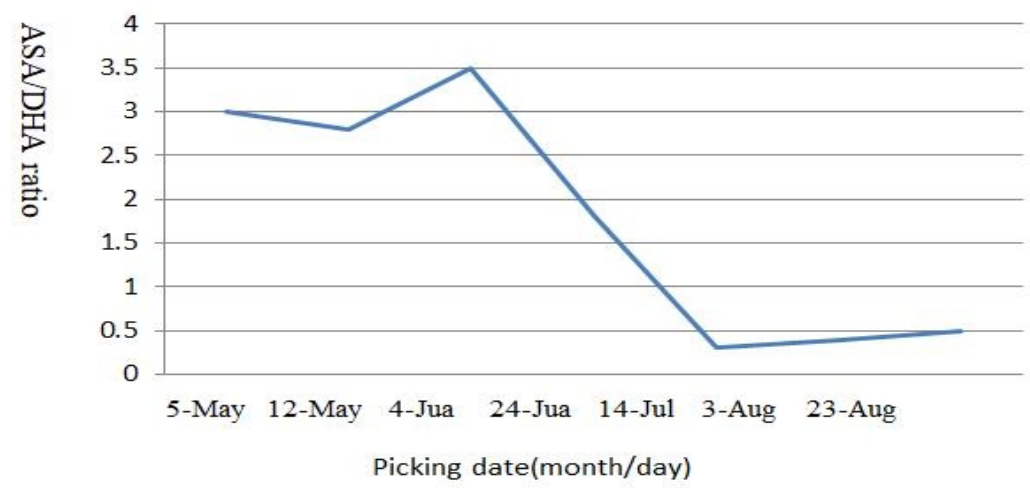

Fig.4, Changes of AsA/DHA content in leaves of Ziziphus jujube 'Zhanshanmizao 1'

Comparison of Ascorbic Acid Content in Different Tissues. As can be seen from Fig.5, ASA content is highest in fruits, followed by leaves, and lowest in flowers. The content of DHA was the highest in leaves, the content in flowers was slightly lower than that in leaves, and the content in fruits was much lower than in leaves. However, the total ascorbic acid content was the highest in the leaves, followed by the fruit, slightly lower than the leaves, and the flower was significantly lower than the other two. Therefore, in the Ziziphus jujube 'Zhanshanmizao 1', the existence of ascorbic acid in fruits, flowers and leaves is quite different, and the total amount is also different.

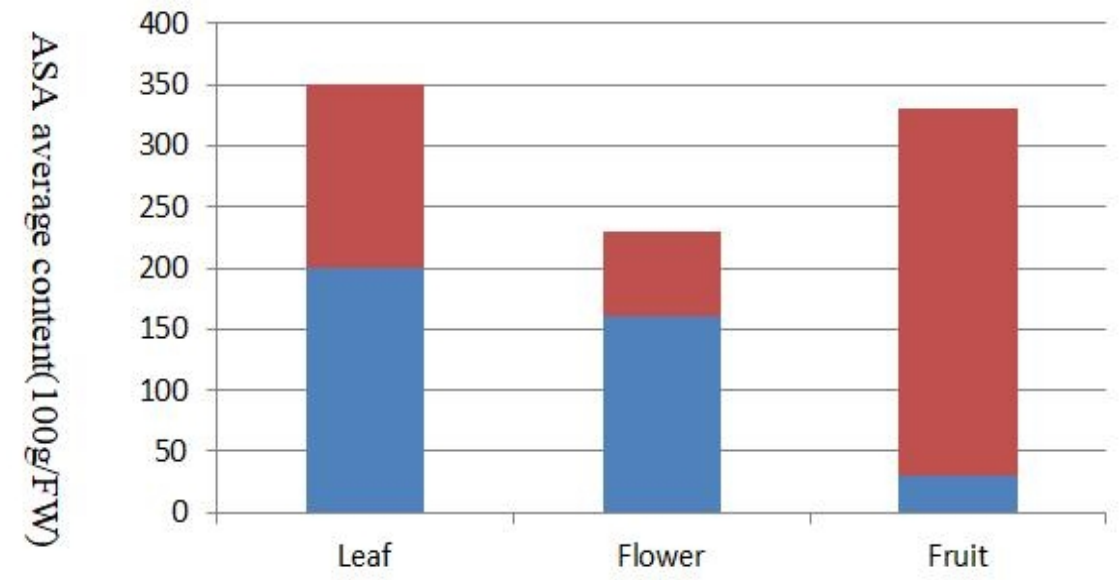

ASA content DHA content

Fig.5, Comparison of ASA and DHA contents in different tissues of Ziziphus jujube 'Zhanshanmizao 1'

\section{Conclusions}

The results of this study showed that ASA content increased rapidly between the green and white maturation stage and reached the maximum at the white maturation stage. With the growth of the leaves, the ASA content in the leaves gradually decreased, and the DHA content decreased first and then increased and drop. The DHA content was basically stable and low in the whole development period. In the same period, there were also significant differences in the form and content of ASA in leaves, fruits and flowers of jujube. DHA content in leaves and flowers accounted for a relatively large proportion, while in fruits, it was mainly ASA; and the total content of leaves was greater than that of fruits and was much larger than that of flowers. In summary, the accumulation rules of leaves and fruits of Ziziphus jujube 'Zhanshanmizao 1' were different, and the ascorbic acid content of different tissues in the same period was different. 


\section{Acknowledgements}

This work was financially supported by the Double subject construction plan of Sichuan Agricultural University and Special action plan project of science and technology rich people and strong county of Sichuan Province "Integration and demonstration of key Technologies for industrialized Development of Zizyphus jujuba 'Zhanshangmizao 1'of Santai County”.

\section{References}

[1] Wang Li, Zhai Anmin, Zhai Zhigang, et al: Effect of Balanced Fertilization on the Yield and Fruit Quality of Mizao Rice Jujube.(thesis of master degree, Western China College of Agriculture and Forestry Sciences,China 2014)

[2] He Lin, Lu Yi, Maoli Xia, Chen Wenhui, et al: Correlation Analysis of Mineral Elements in Soil of Ziziphus jujube`Zhanshanmizao 'and Site. (52)2013,p.1420-142.

[3] Davey MW, Van M, Inzé D, et al: Plant L-ascorbic acid: chemistry, function, metabolism,bioavailability and effects of processing. (80)2000, p. 825-860.

[4] Smirnoff N: The role of active oxygen in the response to water deficit and desiccation. (125)1993,p. 27-58.

[5] Jin Y H, Tao D L, Hao Z Q, et al: Environmental stresses and redox status of ascorbate. (45)2003,p. 795-801.

[6] Gan Lin, Xie Yonghong, Wu Zhengqin, Xia Xianqiang: Changes and Correlation of Vitamin C in Fruit Development of Ziziphus jujuba cv.Jinsixiaozao Journal of Fruit Science. (19)2002,p. 240-242.

[7] Pan Xingjie: Survey of China's Ancient Jujube Resources and Its Utilization. (thesis of master degree, Northwest A\&F University, China 2016).

[8] Wei Tianjun, Dou Yunping: Physiological and Biochemical Changes in the Fruit of Zizyphus jujuba Mill. cv. Lingwuchangzao during its Development and Maturity. (3ed edition, China Agricultural Science Bulletin, China 2008).

[9] Kampfenkel K, Van Montagu M: Inze D.Extraction and determination of ascorbate and dehydroascorbate from plant tissue. Analytical Biochemistry. 1995,225:165-167. 